EOMmun Communication et organisation

Or

Les nouvelles cultures de l'information dans les organisations

\title{
La culture de l'information en milieu universitaire
}

Application à une démarche collaborative de création d'une « start-up communicante »

The information culture in academic circles: Application to a collaborative creation plan for a "communicative start-up"

Smail Khainnar

\section{OpenEdition}

Journals

Édition électronique

URL : http://journals.openedition.org/communicationorganisation/5503

DOI : 10.4000/communicationorganisation.5503

ISBN : 979-10-300-0146-4

ISSN : 1775-3546

Éditeur

Presses universitaires de Bordeaux

Édition imprimée

Date de publication : 1 juin 2017

Pagination : 27-42

ISBN : 979-10-300-0162-4

ISSN : 1168-5549

Référence électronique

Smail Khainnar, "La culture de l'information en milieu universitaire », Communication et organisation

[En ligne], 51 | 2017, mis en ligne le 01 juin 2020, consulté le 02 janvier 2021. URL : http://

journals.openedition.org/communicationorganisation/5503 ; DOl : https://doi.org/10.4000/

communicationorganisation.5503 


\title{
La culture de l'information en milieu universitaire: Application à une démarche collaborative de création d'une «start-up communicante»
}

\author{
Smail Khainnor ${ }^{1}$
}

\section{Introduction}

Notion quadragénaire, la culture de l'information n'a pas cessé de faire couler l'encre des chercheurs de divers horizons scientifiques (sciences de l'éducation, sciences de la bibliothéconomie, sciences de l'information et de la communication, etc.) et des instances internationales (UNESCO, OCDE, Union Européenne, etc.), en se penchant sur divers secteurs (l'école, l'université, l'entreprise, etc.). À ces secteurs d'activité où la formation à l'information est une préoccupation centrale, s'ajoute la problématique citoyenne liée à l'accès à l'information, notamment celle qui concerne les divers scandales (médiator, pilules 3e génération, etc.) (Baltz 2013). Appliquée à un contexte universitaire, la présente étude vise à questionner le «comment » de la mise en place de la notion de culture de l'information en situation pédagogique. Se déroulant dans le cadre d'un module prévu dans la maquette pédagogique d'une formation en cycle master, cette situation englobe des étudiants travaillant en groupe sous l'encadrement d'un enseignant afin de créer une «start-up communicante ». Nous précisons que, au regard de l'orientation organisationnelle du présent dossier thématique (nouvelles cultures de l'information dans les organisations), nous assimilons la situation pédagogique de l'expérimentation (le déroulement des séances du cours) à une organisation. Si nous acceptons avec Jacot qu'une organisation " est un construit humain qui n'a pas de sens en dehors du rapport à ses membres» (Jacot 1991), et avec Mucchielli (Mucchielli 1998) qu'une organisation est une " réalité secondaire ", au sens de l'école de Palo Alto, nous pouvons facilement admettre que le fonctionnement d'une classe (enseignant, étudiants, contenu du cours, coexistence et enchevêtrement de divers contextes régissant les actions entreprises : contexte relationnel social

1 Maître de conférences, Univ Lille Nord de France, F-59000 Lille, France ; UVHC, DeVisu, F-59313 Valenciennes, France. Le Mont Houy. 59313 VALENCIENNES CEDEX 9: smail.khainnar@univvalenciennes. fr 
immédiat, temporel, spatial, physique et sensoriel, juridique et administratif, etc.) n'est autre qu'une forme d'organisation humaine construite par les acteurs de la situation.

Sur un plan rédactionnel, l'article s'organise autour de deux parties. D'ordre théorique et incluant deux réflexions, la première partie se propose d'esquisser, en tenant compte de son caractère pluriel, une délimitation conceptuelle de la culture de l'information. Ainsi, une rapide revue de littérature du concept sera esquissée. Ensuite, en mobilisant une approche situationnelle, une déclinaison pédagogique relative à la formation en culture de l'information dans le monde universitaire sera mise en évidence. À cette première partie théorique, vient s'ajouter une seconde partie empirique visant à mesurer la portée applicative de la culture de l'information en milieu universitaire à travers l'étude d'un fait pédagogique réel. En effet, en adoptant des lentilles situationnelles, nous allons interroger les pratiques informationnelles (recherche, trouvabilité, partage, exploitation, etc.) initiées, conduites et gérées par des étudiants pour créer une entité appelée «start-up communicante » ayant des performances communicationnelles. Une approche qualitative (entretien semi-directif, observation participante, etc.) sera donc convoquée pour analyser lesdites pratiques informationnelles. Les résultats significatifs de cette expérimentation, issus d'une analyse sémantique des réponses recueillies, seront ensuite présentés, discutés et mis en perspective.

\section{Partie théorique: culture de l'information et monde universitaire}

La culture de l'information: une rapide revue de littérature

Datant des années soixante-dix ${ }^{2}$, notamment avec sa définition par l'ALA (American Library Association) en 1989, le concept de culture informationnelle ${ }^{3}$ 'a pas cessé d'être l'objet de divers glissements sémantiques et terminologiques, le rendant ainsi fortement polysémique, voire parfois confus (Serres 2008). Sur un plan historique, trois conceptions ont été forgées autour de cette notion pour en esquisser une délimitation conceptuelle (la conception économique : univers de la veille et de l'intelligence économique, la conception bibliothécaire: habiletés à développer pour survivre dans la société de l'information, et la conception citoyenne: celle relative à la

\footnotetext{
2 Apparition en 1974.

3 La différenciation entre la culture informationnelle et la culture de l'information est rarement formalisée (Liquète, 2014). Plusieurs auteurs se sont emparés de cette question (Baltz 1998), (Fabre et Couzinet 2008), (Serres, 2007, 2008), etc. Globalement, tout le monde est d'accord sur le fait que la première est inclusive de la seconde. En ce qui nous concerne dans le cadre de cet article, nous rejoignions la différenciation faite par Baltz qui, dans un article de Brigitte Simonnot (Simonnot 2009), " propose de distinguer culture informationnelle et culture de l'information, cette dernière étant souvent comprise comme une culture-métier. Il précise d'ailleurs que ce qu'il entend par culture informationnelle pourrait tout à fait se nommer culture communicationnelle pour mieux désigner la synthèse nécessaire à la définition d'un concept qui englobe culture des médias et cyberculture, et qui doit sortir des milieux professionnels pour s'adresser aussi au profane » (Baltz 1998).
} 
vie démocratique) (Le Deuff 2007). Ces conceptions ont laissé apparaître une multitude de discours et déclarations (L'ALA en 1989, L'Ocotillo Information Literacy Group en 1995, L'OQLF4 en 2002, la « Proclamation d'Alexandrie sur la maîtrise de l'information » en 2005, etc.) mettant en exergue un ensemble d'enjeux (éducatifs, socioéconomiques, politiques, etc.) auxquels chaque acteur (politique, citoyen, étudiant, manager, etc.) est censé faire face. S'agissant du concept-même, nous tenons à préciser que la culture de l'information n'est pas strictement un concept documentaire. Il est, comme le précise Juanals (Juanals 2003), un concept « carrefour » se trouvant à la croisée des sciences de l'information et de la communication, des sciences de l'éducation et de l'informatique.

Indépendamment des conceptions, ainsi que les discours et définitions qui en découlent, le concept de culture de l'information peut s'appréhender autour de quatre niveaux s'imbriquant sous la forme d'un étagement. Dans une logique de progression, ces quatre niveaux varient entre le savoir-faire, le savoir, la réflexion critique et la réflexivité (l'autoréflexion). D'ailleurs Shapiro dans un texte fondateur, datant de 1996, considérait déjà ces quatre niveaux en les comparant aux arts libéraux du Moyen-Âge

la culture informationnelle devrait être conçue comme un nouvel art libéral comprenant aussi bien l'utilisation des ordinateurs et l'accès à l'information qu'une réflexion critique sur la nature de l'information elle-même, son infrastructure technique, de même que son impact et son contexte social, culturel et même philosophique. Ces éléments sont considérés aussi essentiels à la structure mentale de l'individu instruit de lère moderne de l'information que le trivium de base des arts libéraux (grammaire, logique et rhétorique) était essentiel à une personne instruite de la société médiévale (Shapiro E̊al. 1996).

Cette définition, qui reste d'actualité dans son aspect général, est, à notre sens, à (re) contextualiser. Cela s'explique par le fait qu'avec les divers développements liés au numérique (web 2.0, web sémantique, RSN, etc.), l'usager, dans sa double posture d' "écritlecteur " 5 selon Broudoux (Broudoux, 2003), n'est pas à l'abri de toutes les formes relatives au phénomène de l'infopollution. Forgé par Sutter (Sutter 1998), ce phénomène se manifeste selon quatre formes : la surabondance, la désinformation, la contamination et les abus publicitaires. C'est ainsi que, en dépassant tout angélisme stipulant que seule une approche d'autodidactie suffise pour affronter la complexité de ces formes d'infopollution, se pose avec acuité la question de la formation de l'usager. Qu'en est-il alors du rôle de la formation en culture de l'information? La section suivante se penche sur cet aspect en deux temps. Il s'agit tout d'abord de présenter d'une manière générale les questions et les enjeux liés à la

4 OQLF: Office Québécois de la Langue Française.

5 Posture impliquant l'usager dans des activités de lecture et/ou d'écriture. 
formation à l'information. Ensuite, en mobilisant une approche situationnelle, un éclairage sera apporté sur le monde universitaire.

La formation en culture de l'information: approche situationnelle du monde universitaire

La problématique de la formation en culture de l'information pose plusieurs questions et enjeux, largement traités par des publications et écrits spécialisés en la matière, que nous essayons de synthétiser ici pour mémoire. S'agissant des questions autour de la formation, en nous appuyant sur les travaux de Serres (Serres 2008), quatre questions peuvent être mises en évidence. Il s'agit, dans un premier temps, de la question didactique relative aux contenus des savoirs entrant dans la construction d'un enseignement de l'information. Ensuite, vient la question des frontières délimitant les trois « territoires » de la culture informationnelle : l'aspect info-documentaire, l'aspect médiatique et l'aspect informatique (formation aux TIC). Cette « question territoriale » vise à s'interroger sur comment partager les formations relatives aux trois aspects. À cela s'ajoute inévitablement la question des acteurs censés assurer les différentes formations, avec la prise en compte de la problématique liée à la gestion du jeu interactionnel se mettant en place entre les trois univers (''infodocumentation, les médias et l'informatique). Enfin, la question pédagogique n'est pas à négliger. Cela concerne la réflexion sur la conception et l'utilisation des supports (notamment avec les TIC) et des designs d'enseignement (modalités et pratiques pédagogiques) à déployer dans les diverses formations (en présentiel ou à distance).

Les enjeux relatifs à la formation en culture de l'information, quant à eux, consistent principalement dans une logique axiologique à s'interroger sur la notion des finalités. Loin d'être tranchées, deux finalités visées par la culture informationnelle peuvent être mises en évidence. D’une part, nous pouvons citer la finalité liée à "l'adaptation ", procédurale et technique, des élèves et des étudiants aux exigences de la nouvelle "société de l'information » et, d'autre part,nous pouvons aussi évoquer la finalité « d'élévation » permettant de former des esprits libres dotés d'une culture critique, y compris contre cette «société de l'information » (Serres 2007). De notre côté, en dépassant tout binarisme opposant les deux visions, nous sommes convaincus de la nécessitéde replacer ces enjeux dans un contexte plus élargi incluant toutes les sphères de la société, notamment la sphère politique. À cet égard, Bernard Stiegler (Stiegler 2005) parle même d'une " politique des technologies de l'esprit " pour mieux penser ces enjeux portant sur le " comment " d'une utilisation intelligente d'internet et des technologies numériques pour augmenter la "valeur esprit ».

Les quatre questions et les deux enjeux relatifs à la formation à l'information ne se déclinent pas de la même façon selon les cibles envisagées (collégiens, 
lycéens, étudiants à l'université, ainsi qu'aux enseignants de ces divers cycles). La nature du public-cible pris dans le cadre de cette étude (étudiants) nous conduit à nous pencher sur le monde universitaire. En effet, la diversité des situations pédagogiques rencontrées dans ce secteur d'activité (apprentissage classique, pédagogie inversée, voire pédagogie renversée ${ }^{6}$, e-learning, $\mathrm{FOAD}^{7}$, etc.) renvoie systématiquement à une variété en matières de modalités de mise en ouvre, de management et d'organisation des pratiques informationnelles mises en place par les acteurs desdites situations d'apprentissage. Ainsi, le concept de «situation » prend ici pleinement son sens. Il conviendrait donc dans un premier temps de s'attarder sur ce concept de "situation ", car notre étude de cas (Cf à la partie applicative, ci-dessous) n'est autre qu'une configuration situationnelle incluant des étudiants œuvrant ensemble, dans le cadre d'un module et sous l'encadrement d'un enseignant, pour créer une «start-up communicante ». Qu'entend-on alors par «situation»?

Dans sa théorie « sémio-contextuelle » de la communication, Mucchielli définit une situation et sa problématique pour un acteur comme « la définition que cet acteur donne de cette situation concrète dans laquelle il est impliqué. Cette situation définit une partie du monde, suffisante pour que les activités immédiates de l'acteur prennent un sens pour lui et lui permettent de tenter de résoudre la problématique posée par cette configuration particulière du monde pour lui » (Mucchielli 2006). Il s'agit donc, comme le précise l'auteur, d'un découpage subjectif du monde par l'acteur pour rendre celui-ci significatif pour lui afin de mieux orienter ses agir en vue de résoudre la problématique posée. En évoquant la volonté de l'acteur de rendre le monde significatif pour lui,la question du « sens » est aussi centrale dans la réflexion sur le concept de "situation ». Cela s'explique par le fait que la situation constitue le contexte principal dans lequel émerge le sens des activités entreprises par l'acteur. Le même auteur précise que le sens naît toujours d'une mise en rapport de quelque chose dans ou par quelque chose. C'est ainsi que l'acteur confronte son activité (discursive, attitudinale, comportementale, etc.) aux éléments significatifs appartenant aux divers contextes ${ }^{8}$ constitutifs de la situation dans laquelle il se trouve pour créer du sens pour lui.

En revenant aux problématiques relatives à la question de l'acquisition des connaissances et des compétences en monde universitaire, nous constatons que les divers acteurs (enseignants, étudiants, etc.) de chaque situation déploient des pratiques informationnelles circonstanciées et situées pour atteindre leurs objectifs (expliquer, former, ou comprendre, assimiler, etc.). Ces pratiques

6 Voir à ce propos les travaux de Jean Charles Cailliez sur la « classe renversée » en biologie moléculaire : http:// blog.educpros.fr/jean-charles-cailliez/a-propos/. Consulté entre 2016/2017.

7 FOAD: Formation Ouverte A Distance.

8 Dans une logique de coprésence et d'enchevêtrement, Mucchielli définit 7 contextes qui composent une situation. Il s'agit du contexte expressif des intentions, projets et enjeux des acteurs en présence, du contexte des normes et règles collectivement partagées, du contexte des positions respectives des acteurs, du contexte relationnel social immédiat, du contexte temporel, du contexte spatial, et du contexte physique et sensoriel. 
dépendent de la façon dont chaque acteur définit la situation (et donc lui donner du sens) dans laquelle il se trouve. Cette (re) construction situationnelle dépend, entre autres, de divers paramètres d'ordre psychocognitif (perception, intuition, représentation, évaluation, décision, etc.) propres à chaque acteur. Ainsi, en relation avec une situation donnée, se déploient diverses activités informationnelles, intentionnelles et/ou passives, pour pouvoir reconnaitre les besoins d'information (explicites, implicites et latents), chercher des informations adéquates à travers un arsenal de techniques (découverte par hasard " sérendipité », navigation, cueillette, équation de recherche, rétroaction pertinente, chaînage, veille, etc.), évaluer et exploiter les informations trouvées dans une perspective solutionniste (supprimer les incertitudes "besoin de connaissance » et/ou modifier les conduites d'activités «besoin d'action » (Le Coadic 1997)). D'ordre applicatif, la partie suivante, en adoptant des lentilles situationnelles, analyse les pratiques informationnelles déployées pardes étudiants universitaires dans un contexte pédagogique.

\section{Partie applicative : la culture de l'information en «situation » pédagogique}

Méthodologie de la recherche

Avant de présenter le cadre méthodologique qui structure la présente étude, nous rappelons que sonobjectif consiste à interroger le " comment " de la mise en place des pratiques informationnelles par des étudiants œuvrant en groupe afin de créer une " start-up communicante ». La nature de l'objectifpoursuivi ici (l'étude d'un fait humain) nous conduit à privilégier une approche d'étude qualitative. Fondées sur un paradigme compréhensif (Paillé 2002 ; Mucchielli 2011), les approches qualitatives visent à atteindre une connaissance intime du phénomène étudié (généralement des phénomènes de sens). Elles permettent aussi de faire surgir les significations profondes construites par la cible (l'étudiant, dans notre cas) au sujet du phénomène étudié (le « comment » de ses pratiques informationnelles partagées avec les coéquipiers de son groupe). Enfin, par la nature de leurs dispositifs de recueil des données (entretiens semi-directifs ou ouverts ou de groupe, observation participante, mise en scène, etc.) et, par conséquent les réponses obtenues, elles échappent à la rigidité qu'un questionnaire fermé peut engendrer (des réponses de conformité et de standing).

C'est ainsi que les données ont été collectées par des entretiens semidirectifs. À cela s'ajoute la mise en place d'une démarche d'observation participante lors des séances du cours. Enfin, des retours ont aussi pu être accueillis lors des exposés oraux, appuyés par des supports informatiques (PowerPoint, etc.), visant à présenter les entités créées. Cela en vue d'une triangulation des données. S'agissant du dépouillement des données recueillies, une méthode d'analyse sémantique a été mobilisée. Dans ce genre d'analyse, 
il s'agit pour le chercheur de procéder, par des opérations et manipulations d'ordre intellectuel, à des transpositions de termes en d'autres termes et des regroupements intuitifs afin de parvenir à déterminer des éléments réduits, homogènes et regroupables. Suite à la mise en évidence de la méthodologie de la recherche, la section suivante se penche sur la présentation du protocole expérimental.

\section{Présentation du protocole expérimental}

Le public-cible pris dans le cadre de notre étude est constitué d'une vingtaine d'étudiants inscrits en première année du master IN2 $\mathrm{C}^{9}$ (Ingénierie Numérique et Collaborative pour la Construction) de l'UVHC (Université de Valenciennes et du Hainaut Cambrésis). L'expérimentation, qui a eu lieu lors du premier semestre ${ }^{10}$ de l'année universitaire 2016/2017, s'est déroulée dans le cadre du module intitulé « communication inter-acteurs » qui a un volume horaire de $12 \mathrm{~h} \mathrm{CM}$ et $6 \mathrm{~h}$ TD. D'un point de vue chronologique, le module s'est organisé autour de 6 séances de 3 h chacune. Pourquoi de tel cours dans un master orienté $\mathrm{BIM}^{11}$ ? En effet, dans les nouvelles démarches collaboratives caractérisant la conduite et la gestion des opérations de construction, la communication, sous diverses formes (immédiate ou médiatée, formelle ou informelle, etc.), constitue une composante clé à la réussite de ces opérations. C'est ainsi que la visée pédagogique de ce module consiste à sensibiliser les étudiants au, d'une part, rôle inévitable et complexe de la communication dans la conduite de projets en BTP et, d'autre part, jeu interactionnel entre la dimension objective d'une opération de construction (mesurabilité, technicité, etc.) et sa dimension subjective (émotivité, conflictualité, etc.). Afin d'atteindre ce double objectif pédagogique, tout en inscrivant les étudiants dans trois postures variant respectivement entre la réception (posture de spectateur), l'application (posture d'acteur) et la création (posture d'auteur), un design d'enseignement en trois séquences a été mis en place. D'un point de vue chronologique, il s'agit des séquences ci-après :

- La séquence de l'étudiant « spectateur » : tout en inscrivant les étudiants dans une position de réception, cette séquence a été mise en place en deux temps. En effet, après avoir communiqué aux étudiants un ensemble d'informations relatif au module (l'objectif pédagogique, la structuration des séances, les modalités d'évaluation, etc.), dans un premier temps, un cours magistral relatifaux liens entre changement organisationnel, communication et émergence du BIM dans le secteur de la construction,

9 Nous rappelons que cette promotion constitue la première promotion de ce master qui a ouvert durant cette année $(2016 / 2017)$.

10 Le premier semestre de cette formation a eu lieu entre septembre 2016 et fin janvier 2017.

11 BIM: Building Information Model, ou Modeling ou Management. Diverses interprétations sont possibles selon les contextes d'usage de l'acronyme. 
a eu lieu, dans un second temps. Ainsi, le contenu théorique communiqué permet aux étudiants de mieux penser et accompagner les futures évolutions et réorganisations du secteur de la construction générées par la mise en place du BIM;

- La séquence de l'étudiant "acteur » : placé dans une posture favorisant l'action et en procédant par une mobilisation notionnelle du contenu du cours magistral, chaque étudiant rédige une synthèse portant sur l'analyse des pratiques communicationnelles de son établissement d'accuei ${ }^{12}$. Cette synthèse aborde les points forts et les points faibles de modes actuels de communication (interne et externe) de l'établissement d'accueil, tout en proposant des pistes d'amélioration aux carences communicationnelles relevées ;

- La séquence de l'étudiant « auteur » : réunis en groupes et s'inscrivant dans une logique de liberté créatrice, les étudiants créent une entité appelée «start-up communicante» ayant des performances communicationnelles. Ces performances se nourrissent, d'une part, des apports théoriques du cours magistral et, d'autre part, du croisement des points forts de la communication de divers établissements d'accueil relevés dans le cadre des synthèses individuelles élaborées par chaque étudiant. Des exposés de $30 \mathrm{mn}$ chacun (15 $\mathrm{mn}$ de présentation suivie par $15 \mathrm{mn}$ d'échange et de débat) ont été mis en place à la fin du module pour permettre aux différents groupes de présenter leur création. À l'issue de cet exercice créatif, une synthèse finale a été rédigée par chaque groupe. Qualifiée de " méta-communicationnelle ", cette synthèse porte sur deux aspects. D'un côté, chaque groupe est censé, avec du recul, analyser ses pratiques communicationnelles servant à la création de son entité (de la genèse de l'idée jusqu'à l'exposé final et les réponses aux questions) et, de l'autre, analyser l'évolution de son regard sur le BIM après quelques mois de formation en master IN2C.

À la fin du module, toutes les données ont été récupérées par l'enseignant. Pour rappel, il s'agit des données issues des entretiens semi-directifs, des données issues de lobservation participante, des données sous forme de notes prises par l'enseignant lors des exposés oraux avec la récupération des supports PowerPoint de chaque groupe, et les données du premier volet de l'analyse méta-communicationnelle (l'analyse des pratiques communicationnelles intra et inter groupes pour créer la "start-up communicante »). Ainsi, le travail de sémantisation commence.

12 Nous rappelons que les étudiants du master IN2C sont inscrits en contrat de professionnalisation et en formation continue. À cet égard, ils alternent entre des périodes de cours à l'université et des sessions d'apprentissage chez divers établissements d'accueil (BET, entreprises, prescripteurs de matériaux de construction, etc.) utilisant, ou vont utiliser, le BIM. 


\section{Présentation et discussion des résultats}

À l'issue du dépouillement des données collectées, les pratiques informationnelles mises en place par les étudiants pour la création de la « start-up communicante " ont été regroupées sémantiquement en trois catégories. Chacune d'entre elles met en évidence l'émergence, le développement et l'ancrage d'une micro-culture informationnelle caractérisant une facette particulière du travail collectif engagé par les divers groupes de la promotion. Par micro-culture informationnelle, nous entendons un ensemble de conduites (cognitives, expressives, attitudinales, comportementales, etc.) qui émerge, à une échelle intra et/ou inter individuelle, dans et pour la situation de la démarche collective. Cet ensemble émergent se développe et s'ancre progressivement dans les pratiques des étudiants, et finit par avoir un statut d'une autorité normative cadrant leur agir (donner suite aux messages envoyés par les autres, se montrer réactif dans le suivi et l'accompagnement des changements touchant le travail de groupe, etc.). Ainsi, nous pouvons distinguer les micro-cultures ci-après :

- micro-culture identificationnelle: portant sur le «qui », cette micro-culture concerne toutes les pratiques informationnelles mises en place par les membres de chaque groupe, notamment en amont de la démarche collective, pour s'identifier mutuellement. Il s'agit dans ce sens du besoin des étudiants d'avoir une connaissance mutuelle incluant le soi-même (ses compétences, ses faiblesses, ses souhaits, etc.) et l'autre (ses études antérieures, son secteur d'activité au sein de l'établissement d'accueil de son apprentissage, ses compétences en informatique, etc.). Cela pour mieux organiser et coordonner les efforts afin d'optimiser la création de l'entité demandée. Ainsi, diverses pratiques informationnelles (des tours de table pour se présenter, des confrontations de divers points de vue relatifs à la conception de l'exercice créatif, le croisement des analyses des pratiques communicationnelles des établissements d'accueil élaborées individuellement (Cf. à la séquence de l'étudiant " acteur », etc.) ont été mises en place pour mieux se connaître afin d'avancer efficacement. Ce besoin de se connaître mutuellement révèle en son sein un autre besoin, implicitement exprimé, à savoir celui de la confiance. Maintes fois est revenue dans les expressions des étudiants la nécessité de se faire confiance et de travailler en confiance. La confiance, comme concept récursif (Processus et Résultat, selon Le Moigne 1991), apparaît donc ici un ingrédient majeur aux yeux des étudiants, ce qui les a amenés à partager les diverses informations possédées afin de parvenir à une construction collective négociée de la création de la «start-up communicante».

- micro-culture processuelle : portant sur le «comment», cette micro-culture concerne les pratiques informationnelles déployées par les étudiants afin de concevoir, conduire et gérer le processus de création de l'entité 
demandée. L'analyse des réponses des étudiants, à propos de leurs façons d'agir, révèle la possibilité d'une modélisation normative d'un processustype commun à tous les groupes. S'inspirant du modèle du "Double Diamant », conçu en 2005 par le Design Council (Royaume-Uni), ce processus-type se présente comme la succussion de deux grandes étapes, ayant chacune deux sous-étapes. Comme première étape, les étudiants ont commencé par vouloir s'imprégner du sujet de l'exercice créatif demandé. Pour ce faire, ils ont, tout d'abord, passé par une sous-étape de Découverte visant à chercher des informations leur permettant de mieux comprendre les différents ingrédients du sujet (communication, notion de start-up, analyse des cas, etc.). Ensuite, ils ont fini par une sousétape de Défnition d'une trame conceptuelle de leur future «start-up communicante » (secteur d'activité, logo, caractéristiques fonctionnelles, organisationnelles, etc.). La deuxième étape, quant à elle, a commencé par une sous-étape de Développement visant, dans une logique de réouverture, à concevoir et à créer la « start-up communicante » proprement dite. Ainsi, toutes les caractéristiques (organisationnelles, fonctionnelles, structurelles, etc.) ont été définies. Enfin, sous forme d'une (Dé)13 livraison, la dernière sous-étape a consisté, suite aux échanges avec l'enseignant, à valider les choix et les partis du produit « start-up communicante ". Ce modèle quasi-canonique, qui s'est cristallisé à travers les pratiques informationnelles des différents groupes, met en évidence la capacité des étudiants à faire face à l'infobésité caractérisant leur environnement de travail. Cela se concrétise par la mise en œuvre d'une logique d' " aiguillage informationnel » leur permettant, dans un premier temps, de naviguer dans des gisements informationnels, et par la suite, d'en sortir avec des informations sélectionnées en adéquation avec la nature du problème à résoudre. La figure 1 , ci-dessous, présente le modèle du Double Diamant commenté par l'auteur.

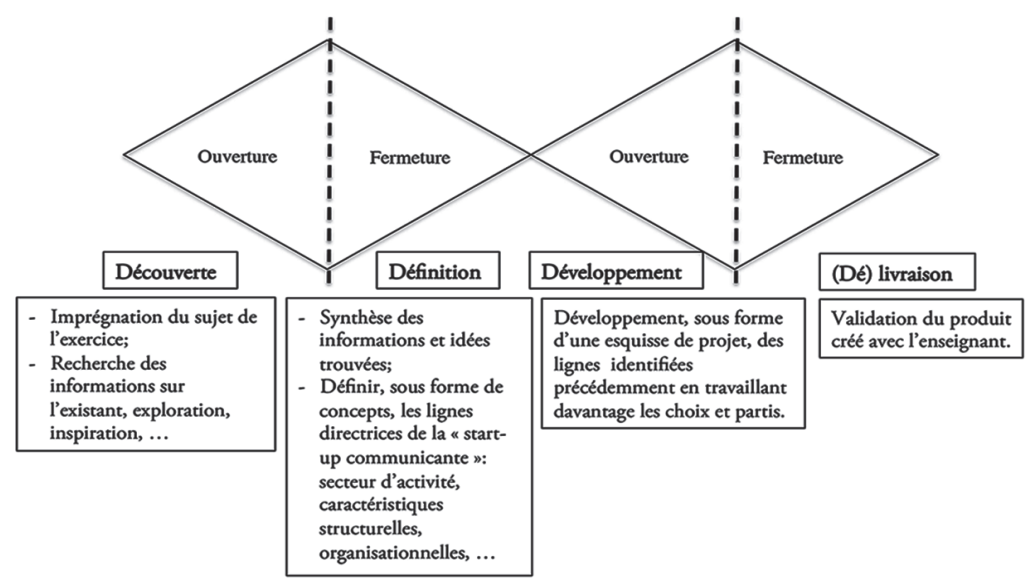

Figure 1: La micro-culture processuelle vue avec le prisme du modèle «Double Diamant » commenté par l'auteur. 
- micro-culture contextuelle : portant sur le « où » se passe, cette microculture fait référence à toutes les pratiques informationnelles mises en place par les étudiants pour prendre en compte les ingrédients de l'environnement du travail dans lequel ils se trouvent. En effet, divers paramètres ont contribué à complexifier le travail collectif des étudiants (l'alternance entre les sessions d'apprentissage dans les établissements d'accueil et les sessions de formation à l'université, les autres modules de la formation à gérer (notamment ceux qui concernent l'aspect purement informatique, vu la nature du master poursuivi), les vacances du Noel, etc.). Cela a inscrit les étudiants dans une logique d'agir atemporelle pour pouvoir avancer efficacement sur leur création indépendamment du calendrier pédagogique de leur semestre. Ainsi, grâce essentiellement aux potentialités communicationnelles des TIC, diverses pratiques partagées de l'information ont été mises en place pour faire face aux contraintes contextuelles. Mis à part les pratiques informationnelles d'ordre présentiel (séances de travail en groupe lors des séances du cours, à la bibliothèque universitaire, etc.), l'analyse des réponses des étudiants met en évidence la mobilisation de deux types de pratiques informationnelles d'ordre distantiel. Il s'agit, d'une part, des pratiques que nous qualifions de "discursives ", concrétisées principalement par des visioconférences visant à transmettre oralement les nouvelles informations et faire des points réguliers sur l'état d'avancement de l'exercice créatif et, d'autre part, des pratiques que nous qualifions de " consultatives ", concrétisées par la création de divers comptes afin de stocker et de consulter les informations (compte sur Google Drive, etc.). Cette adaptabilité situationnelle, quant aux pratiques informationnelles déployées (pratiques orales, de lecture, d'écriture, de mise en partage, etc.), témoigne de la sensibilité systémique ${ }^{14}$ chez les étudiants qui, en prenant en compte tous les paramètres de la situation, essayent d'avancer tout en priorisant leurs actions.

L'interaction entre les trois micro-cultures, mises en évidence ci-dessus, donne lieu à une culture de l'information que nous qualifions de " collaborative ». Le fait d'évoquer l'aspect collaboratif s'explique par le fait que, contrairement aux processus coopératifs ${ }^{15}$, les étudiants de chaque groupe, impliqués dans un engagement mutuel, ont œuvré ensemble dans une logique solidaire pour répondre au cahier des charges de l'exercice pédagogique demandé (création de la «start-up communicante »). L'engagement mutuel et l'agir solidaire de chaque groupe ont permis la cristallisation des trois micro-cultures. Ainsi,

14 Nous rappelons que la « systémique » est un concept central dans le cours magistral administré aux étudiants lors de la première séquence pédagogique de létudiant « spectateur » (Cfà la section : présentation du protocole expérimental).

15 La cooperation consiste à juxtaposer les efforts des acteurs, agissant d'une manière autonome, pour réunir à la fin leurs contributions en vue d'atteindre l'objectif initialement fixé. (Piquet 2009). 
Loin d'être une donnée de la situation (un " déjà-là » qui s'imposait aux étudiants dès le début de l'exercice), les trois micro-cultures constituent donc une co-construction (une émergence) mises en place par et pour eux afin de répondre d'une manière située à la problématique posée. En convoquant un double raisonnement, à la fois géométrique (conception circulaire) et matriciel (tableau), nous considérons alors la « culture de l'information collaborative » comme la résultante de l'émergence des trois micro-cultures, où chacune est représentée par une portion du cercle (figure 2).

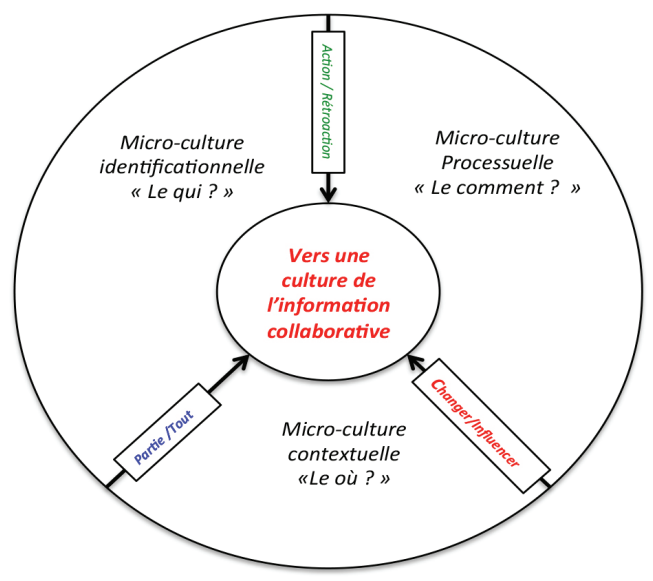

Figure 2 : Modélisation géométrique des résultats de l'étude. Source : auteur

Enfin, l'interaction entre chaque couple de micro-culture est explicitée par un rectangle incluant deux expressions (mots ou verbes). En respectant le même langage couleur utilisé dans la figure de la modélisation géométrique des résultats, ci-dessus, ces interactions sont explicitées sur le tableau 1 ci-après :

\begin{tabular}{|c|c|c|c|}
\hline & $\begin{array}{c}\text { Micro-culture } \\
\text { identificationnelle }\end{array}$ & $\begin{array}{l}\text { Micro-culture } \\
\text { processuelle }\end{array}$ & Micro-culture contextuelle \\
\hline $\begin{array}{l}\text { Micro-culture } \\
\text { identificationnelle }\end{array}$ & & $\begin{array}{l}\text { Action: du «qui » vers le } \\
\text { «comment»: les acteurs } \\
\text { fabriquent et agissent } \\
\text { sur le processus. }\end{array}$ & $\begin{array}{l}\text { Partie: du «qui» vers } \\
\text { le «où»: les acteurs } \\
\text { constituent une partie du } \\
\text { système englobant dans } \\
\text { lequel ils agissent. }\end{array}$ \\
\hline $\begin{array}{l}\text { Micro-culture } \\
\text { processuelle }\end{array}$ & $\begin{array}{l}\text { Rétroaction : du } \\
\text { "comment» vers le } \\
\text { "qui»: le processus } \\
\text { exerce, à son tour, } \\
\text { un feed-back sur les } \\
\text { actions entreprises par } \\
\text { les acteurs. }\end{array}$ & & $\begin{array}{l}\text { Changer : du « comment } \\
\text { vers le " où » : le processus } \\
\text { produit des changements } \\
\text { dans l'environnement dans } \\
\text { lequel il se produit. }\end{array}$ \\
\hline
\end{tabular}




\begin{tabular}{|c|c|c|}
\hline $\begin{array}{l}\text { Micro-culture } \\
\text { contextuelle }\end{array}$ & $\begin{array}{l}\text { Tout : du « où » vers } \\
\text { le « qui »: le contexte } \\
\text { constitue un tout } \\
\text { dans lequel les acteurs } \\
\text { forment une partie } \\
\text { agissante. }\end{array}$ & $\begin{array}{l}\text { Influencer: du « où " } \\
\text { vers le « comment ": } \\
\text { le contexte influe sur } \\
\text { la conception et le } \\
\text { fonctionnement du } \\
\text { processus. }\end{array}$ \\
\hline
\end{tabular}

Tableau 1 : Explicitation des interactions entre les trois micro-cultures. Source : auteur.

\section{Conclusion et perspectives}

Lobjectif de cette étude a été de questionner le "comment " de la mise en place des pratiques partagées de l'information par des étudiants dans une situation pédagogique. Pour ce faire, deux parties, théorique et applicative, se sont succédé pour structurer l'article. Ainsi, la première partie est revenue, dans un premier temps, sur une rapide revue de littérature relative au concept de culture de l'information, pour ensuite, en mobilisant une approche situationnelle, traiter la question liée à la formation à l'information en monde universitaire.En convoquant une approche d'étude qualitative, les pratiques informationnelles des étudiants, réunis en groupes pour créer une " startup communicante " dans le cadre de l'un des modules de leur formation, ont été analysées dans une seconde partie applicative. Trois micro-cultures informationnelles, s'articulant systémiquement, ont donc été dégagées à l'issue du dépouillement des données recueillies. Variant entre « le qui », « le comment » et « le où » (Acteur-Processus-Contexte), ces trois micro-cultures inscrivent les agir des étudiants dans une logique de « culture de l'information collaborative ». La cristallisation des trois micro-cultures, à travers le design d'enseignement mis en place dans le cadre de l'expérimentation, livre de riches enseignements sur le métier de l'étudiant, sur celui de l'enseignant et, par conséquent et plus largement, sur la relation à l'information dans le monde universitaire.

Tout d'abord et s'agissant de l'étudiant, nous pouvons dire que le caractère transmissif-applicatif-créatif de notre design d'enseignement, avec ses trois séquences pédagogiques (spectateur, acteur et auteur), a pu révéler l'efficacité de la gestion informationnelle menée par les étudiants dans la création de l'entité demandée. Ainsi, leurs pratiques partagées de l'information déployées les ont inscrits dans une nouvelle posture que nous qualifions d' " étudiant-entrepreneur ", plutôt que la posture classique d'un simple étudiant-récepteur. Selon Verstraete (Verstraete 2000), la modélisation de l'activité entrepreneuriale met en évidence l'existence de trois dimensions : la dimension cognitive (la pensée et les connaissances), la dimension praxéologique (l'action) et la dimension structurale (la structure). Cela se transpose parfaitement au cas de nos étudiants-entrepreneurs qui agissaient cognitivement d'une manière structurée. Ensuite et s'agissant de l'enseignant, 
au-delà de son rôle classique d'un transmetteur de savoir, il est devenu dans le cadre du design d'enseignement de l'expérimentation un " connecteur de compétences ». Comme un designer, la tâche de l'enseignant dans ce cadre a été, dans une logique pensante et orientée vers le futur, de canaliser et optimiser les intentions mélioratives de divers groupes dans la création de leur «startup communicante ». Enfin, s'agissant de la relation àla conception et l'usage de l'information, nous pouvons considérer que cette dernière est devenue une ressource à part entière dans le processus d'apprentissage. Elle n'est plus un produit transmis par l'enseignant à l'étudiant dans un rapport au savoir asymétrique. Elle est plutôt le ciment liant les deux acteurs (enseignant et étudiant) dans l'écosystème de la communication scientifique (Broudoux 2013). En guise de perspectives à cette étude, à la lumière des incidences mises en exergue ici sur la trilogie enseignant-étudiant-rapport à l'information, nous pouvons évoquer l'importance d'esquisser les nouveaux rôles de l'université de demain. La RSU (Responsabilité Sociale des Universités), qui a déjà commencé à se profiler depuis quelques années, peut, à cet égard, constituer un cadre pertinent pour nos futures analyses et recherches sur le sujet.

\section{BIBLIOGRAPHIE}

BALTZ Claude, 2013, "Meurtre de l'information ? ... ", in Pratiques et usages numériques, H2PTM'13, Paris, Édition Lavoisier, p. 3-12.

BALTZ Claude, 1998, "Une culture pour la société de l'information ? Position théorique, définition, enjeux ", Documentaliste - Sciences de l'information, vol. 35, $\mathrm{n}^{\circ} 2$, p. 75-82.

BROUDOUX Evelyne, 2003, Autoritativité, support informatique, mémoire.Journées hypertextes, mémoire, fiction. Montréal. Novembre. Voir rectification ci-dessous pour confirmation.

BROUDOUX Évelyne, 2003, "Autoritativité, support informatique, mémoire ", Archive ouverte en sciences de l'information et de la communication, @ rchivesic 2003 [disponible sur https://archivesic.ccsd.cnrs.fr/sic_00001137

BROUDOUX Evelyne, 2013, L'écosystème scientifique à l'heure de la participation sur le web. In Pratiques et usages numériques H2PTM'13. Édition Lavoisier. ISBN : 9782746245792.

FABRE Isabelle,COUZINETViviane,2008,Désir,curiosité,culture informationnelle: l'organisation des savoirs au cœur de l'histoire des idées, in Revue Canadienne des sciences de l'information et de la bibliothéconomie, vol. 32, p. 85-105. <hal-00802749>

JACOT Jacques Henri (dir), 1994, Formes anciennes, formes nouvelles d'organisation, Presses Universitaires de Lyon.

JUANALS Brigitte, 2003, La culture de l'information. Du livre au numérique, Paris, Édition Lavoisier.

LE COADIC Yves François, 1997, Les Sciences de l'information, Édition Que-sais-je ? 
LE DEUFF Olivier, 2007, La culture de l'information : Quelles « littératies » pour quelles conceptions de l'information? In VI ${ }^{\mathrm{e}}$ Colloque ISKO-France 2007, 7 et 8 juin. Toulouse, IUT de l'Université Paul Sabatier, Toulouse.

LE MOIGNE Jean Louis, 1991, Script d'une conférence donnée au colloque « Du Mépris à la Confiance, de nouveaux comportements pour faire face à la complexité » organisée par l'Université de Technologie de Compiègne (TSH/IDTH), sous la direction de G. Le Cardinal et de J. F. Guyonnet.

LIQUETE Vincent (coord.), 2014, La culture de l'information. Paris : CNRS éd. (Les essentiels).

MUCCHIELLI Alex, 2011, Dictionnaire des méthodes qualitatives en sciences humaines. 3e Édition Armand Colin.

MUCCHIELLI Alex, 2006, Étude des communications : Nouvelles approches, Paris, Édition Armand Colin, Collection U, 220 pages.

MUCCHIELLI Alex, 1998, Approche systémique et communicationnelle des organisations, Paris, Édition Armand Colin, Collection sciences de la communication, p. 160.

PAILLE Pierre $\Xi$ al, 2002, L'analyse qualitative, Paris, Édition Armand Colin.

PIQUET Alexandre, 2009, "Guide pratique du travail collaboratif : Théories, méthodes et outils au service de la collaboration», Document destiné au "Groupe Communication » du réseau Isolement Social, Brest. Disponible sur http://www.a-brest. net/IMG/pdf/Guide_pratique_du_travail_collaboratif.pdf

SERRES Alexandre, 2008, La culture informationnelle, in Problématiques émergentes dans les sciences de l'information (sous la direction de Fabrice Papy), Lavoisier, p. 137-160.

SERRES Alexandre, 2007, Questions autour de la culture informationnelle, La Revue canadienne des sciences de l'information et de bibliothéconomie, vol. 31, n 1, p. 69-85.

SIMONNOT Brigitte, 2009, « Culture informationnelle, culture numérique : au-delà de l'utilitaire ", in Cabiers du numérique, 2009/3, vol. 5, p. 25-37.

STIEGLER Bernard et Ars Industrialis, 2006, Réenchanter le monde- La valeur esprit contre le populisme industriel, Paris, Édition Flammarion, 172 p.

SHAPIRO Jeremy, SHELLEY K. Hughes, 1996, Information Literacy as a Liberal Art, Educom Review, vol. 31, no. 2 March/April.

SUTTER Éric, 1998, « Pour une écologie de l'information », Documentaliste-Sciences de l'information, vol. 35, 2, p. 83-86.

VERSTRAETE Thierry, 2000, Entrepreneuriat en sciences de gestion. HDR en sciences de gestion. Université de Lille 1. Disponible sur http://thierry-verstraete.com/pdf/Hdr tv.pdf

Résumé : Cet article vise à interroger le « comment » de la mise en place de la culture de l'information dans une situation pédagogique. Il s'attache à montrer, à travers une étude de cas portant sur la création d'une "start-up communicante " par des étudiants universitaires, les incidences de leurs pratiques partagées de l'information sur les modalités de mise en œuvre, de management et d'organisation de leur communication. 
Mots-clés : culture de l'information, monde universitaire, étudiant, apprentissage, situation.

Abstract: This article aims to examine how the information literacy is built in a pedagogical situation. It attaches to show, through a case study where university students create an entity called «start-up communicante», the consequences of their shared practices of the information on the management and the organization of their communication.

Keywords: information literacy, the academic world, student, learning, situation. 